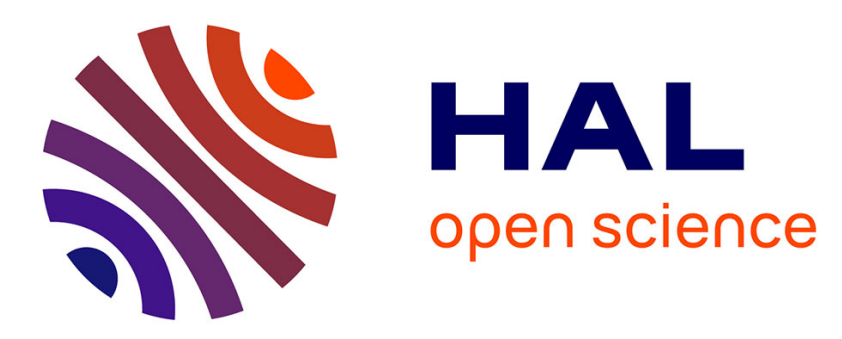

\title{
Between two engineering ages: Of information and complex systems, professional research is based on a team work!
}

Alexandru Serbanescu, Adrian Diaconu, Cornel Ioana, Angela Digulescu

\section{To cite this version:}

Alexandru Serbanescu, Adrian Diaconu, Cornel Ioana, Angela Digulescu. Between two engineering ages: Of information and complex systems, professional research is based on a team work!. ECAI 2015 - 7th International Conference on Electronics, Computers and Artificial Intelligence, Jun 2015, Bucharest, Romania. 10.1109/ECAI.2015.7301136 . hal-01448221

\section{HAL Id: hal-01448221 \\ https://hal.science/hal-01448221}

Submitted on 27 Jan 2017

HAL is a multi-disciplinary open access archive for the deposit and dissemination of scientific research documents, whether they are published or not. The documents may come from teaching and research institutions in France or abroad, or from public or private research centers.
L'archive ouverte pluridisciplinaire $\mathbf{H A L}$, est destinée au dépôt et à la diffusion de documents scientifiques de niveau recherche, publiés ou non, émanant des établissements d'enseignement et de recherche français ou étrangers, des laboratoires publics ou privés. 


\section{Between Two Engineering Ages: Of Information And Complex Systems,}

\section{Profesional Research Is Based On A Team Work!}

\author{
Alexandru SERBANESCU ${ }^{1,2}$, Adrian \\ DIACONU ${ }^{2}$ \\ ${ }^{1}$ Military Technical Academy \\ 2"LUMINA"- The University of South-East Europe \\ Bucharest, Romania \\ alexe1serbanescu@yahoo.com
}

\author{
Cornel IOANA ${ }^{3}$, Angela DIGULESCU ${ }^{1,3}$ \\ ${ }^{3}$ GIPSA-lab, Grenoble Institute of Technology \\ Grenoble, France \\ angela.digulescu@gipsa-lab.grenoble-inp.fr \\ cornel.ioana@gipsa-lab.grenoble-inp.fr
}

\begin{abstract}
From twenty years we promote the idea that engineering's systems (and not only!) are nonlinear ones and usually can have chaotically behaviors and their associated processes need special studies' methods. In this paper we present two directions from our research: cryptography based on chaos and a new method to analyze signals from nonlinear dynamical systems.
\end{abstract}

Keywords - Nonlinear complex systems, hinking in systems, cryptography based on chaos, signal processing based on recurrence plot ananlysis.

In its most general sense, engineering is turning an idea into a reality, creating and using tools to accomplish a task or fulfill a purpose. There is the appreciation that the story of engineering divides into five ages: gravity, heat, electromagnetism, information, and systems. The same specialists appreciate that we are in the age of information, which has turned now into the age of complex systems.

Complexity describes objects with many interconnected parts and complex systems are a subfield of computer science, called computational complexity. Speaking of the concepts: complicated and complex we can say that it is easy to distinguish one from the other only at the extremes, but there is a middle ground where the distinction becomes unclear and arbitrary. The interactions of interest are nonlinear and this non-linearity yields levels of organization and hierarchies. Complex systems exhibit several kinds of behavior such as: selforganization into patterns, chaotic behavior adaptive interaction.

Analyzing complexity, we can ask how systems can generate perpetual novelty using limited resources. And besides many answers two concepts become important: laws and states. For example, for a system, the course over time appears chaotic rather than deterministic. Though the partial differential equations describing chaotic systems are fully deterministic, the presumed guarantees of determinism: similar starting conditions yield similar state trajectories - no longer hold!
We must take in account a systemic approach because a system is more than the sum of its parts. It may exhibit adaptive, dynamic, goal-seeking, selfpreserving, and sometimes evolutionary behavior. The least obvious part of the system, its function or purpose, is often the most crucial determinant of the system's behavior. Complex behaviors of systems often arise as the relative strengths of feedback loops shift, causing first one loop and then another to dominate behavior! What can we do?

Today, many authors recommend: Listen to the "Wisdom of the Systems", stay humble - Stay a Learner!

Working with systems, often reminds me of how incomplete my mental models are, how complex the world is, and how much I don't know! Sometimes, I think to defy the Disciplines because seeing systems whole requires even more than being "interdisciplinary". Even so, we will contemplate the inevitable gaps between what we know, what we do, and why things go wrong. But, as we explore modern ideas of systems complexity, we will see that the gaps are filled by risk. Risk is at the heart of major engineering questions. How do we know what is safe? How safe is safe enough?

The simplest way of minimizing the risk of failure of a physical 'hard' tool is to use a large safety factor, but some tools have become so very complex.

Engineers have started to work and manage the risks in layers or levels. Strictly specializations in IT\&C, for example, pose new risks since the relationships between levels and, more importantly, between the ways of understanding of specialists in those levels, are not straightforward.

Now, in the 21 st century, we are entering into the age of systems with a potential for new risks through interdependencies we may not fully understand. For example, we now know that some (but not all) physical processes are chaotic, in the sense that, whilst they may appear to be reasonably simple, they are inherently difficult to predict. We have discovered that they may be very sensitive to very small 
differences in initial conditions and may contain points of instability where paths diverge.

Energy, entropy, and exergy are examples of how the challenges of the $21 \mathrm{st}$ century require the engineering disciplines that are much better at integrating their expertise to find synergy. As a result, some engineers have begun to think differently - they use what many call 'systems thinking' and an engineering team is an example of a specific soft system.

If we are to make and maintain highly reliable and sustainable complex systems, then we need more of our specialist engineers to be systems thinkers that can deal both with the detail and the big picture - a synergy from the integration of reductionism and holism.

Speaking about our research activity, I can confess that it was influenced by scientific trends, by our professors, our colleges and in the last thirty years by our enthusiastic $\mathrm{PhD}$ students.

In the following sections we present, for example, two directions from our research: cryptography based on chaos and a new method to analyze signals from nonlinear dynamical systems.

\section{IMAGE ENCRYPTION ALGORITHMS BASED ON GAMES' THEORY}

Within the past few years a new trend in the field of digital images' cryptographic algorithms' designing branch has been revealed. A new-built and unique approach (targeting the fruitful conjunction between games' theory, namely their rules' design principles, and cryptography, be it either classical or chaosbased), which would crystallize in its early years, was identified. Among the games whose principles are used in the designing of cryptographic algorithm can be enumerated, e.g., Rubik's cube [1], Sudoku [2], Chinese Chess [3] etc.

An extended improved version of an image encryption algorithm based on Rubik's cube principle and digital chaotic map is presented in [1]. Basically, as shown in Fig. 1, through an iterative process, image's rows and columns are scrambled via cyclic rotations (either to the left or right, resp., up or down, with a random number of steps, as determined by the intrinsic properties of the image). Then, by the use of a chaotic map, image pixels are doubly ciphered.

Other ideas revealed within the previously mentioned paper were: (i) to relocate original images' pixels as dictated by the associated Sudoku gird (i.e., constructed with the same dimensions as of image to be scrambled and (or) ciphered), as Fig. 2 suggests [2]; (ii) to take advantage of Knight's moving pattern nonlinearity in order to change image pixels' position both spatially and between the RGB channels.

Within their research works, with the aid of standard analyses (e.g., statistical - histogram, adjacent pixels' correlation coefficients; differential NPCR and UACI indices; local and global entropy; key space and key sensitivity to small changes; strength to different types of attacks etc.) authors of the above mentioned papers have proved that the newly proposed digital images' encryption schemes have similar or better performances with those proposed by other scholars.

As easily it can be seen, all this was possible only through the combined efforts of an intricate network of enthusiastic scholars with common research interests.

Figure 1. Image scrambling using Rubik's cube principle. Here, $\mathrm{I}(\mathrm{i}, \mathrm{j})$ represents image's pixels, $\beta_{1}$ and $\beta_{2}$ are two random integers (which subsequently will be part of the encryption key) and 'mod' represents the modulo operator.

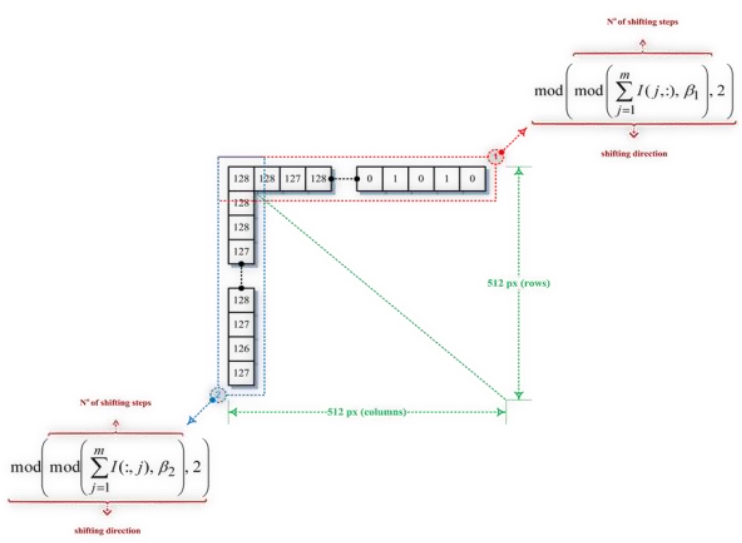

Figure 2. Inter bit-level permutation: (a) input image's associated matrix, (b) the $S G_{256}$ matrix, (c) stage's intermediate output image (i.e., with only two of $1^{\text {st }}$ row's pixels relocated).

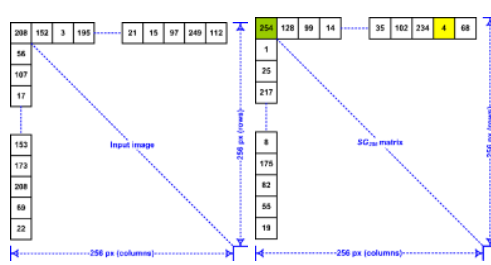

(a)

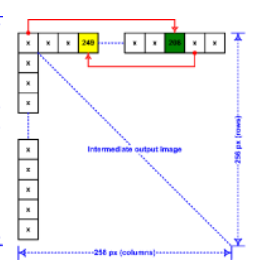

(c)

\section{NEW APPLICATIONS OF TRANSIENT SIGNAL PROCESSING}

Transient signals are signals with short duration and broad spectrum which are often present in various industrial applications such as turbine regime changes, electrical arches generated in photovoltaic panels or in electric cables, etc. In order to ensure the safety of such systems, it is very important that these signals can be detected, located and characterized in order to prevent an eventual irreversible damage. This paper presents new tools derived from the concept of Recurrence Plot Analysis (RPA) and applied to some real applications related to power systems. The concept of RPA is presented in figure 3.

Our applications concern the detection, localization and characterization of the transient signals measured in hydrodynamic systems and in electrical systems, respectively. The applications for the hydrodynamic systems particularly refer to the detection and quantification of the water hammer effect and to the Rankine vortex rope detection, while the electrical systems applications refer to the detection, localization and characterization of partial 
discharges in high voltage cables and of electrical arcs that appear in photovoltaic panels, respectively.

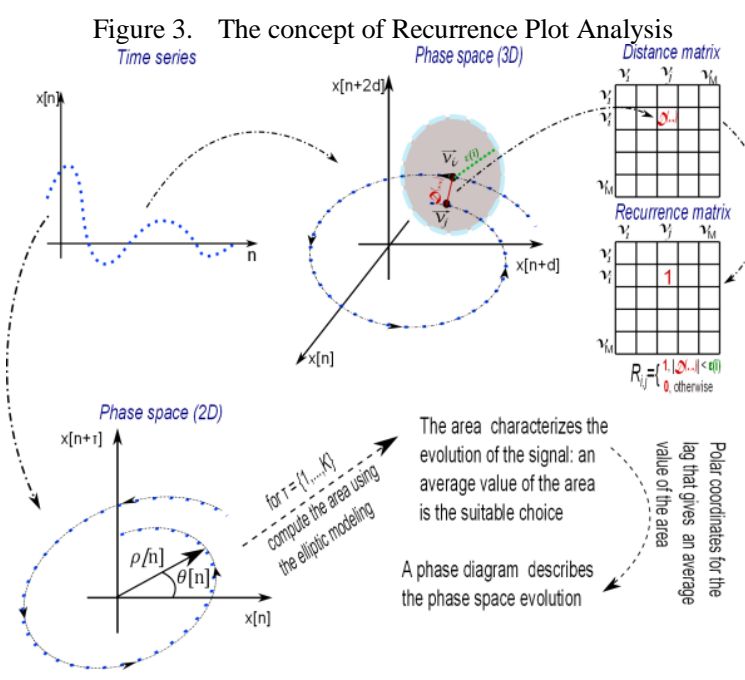

For the detection and localization part, we used the multi-dimensional representation and its corresponding recurrence matrix for the computation of the TDR - time distributed recurrence measure:

$$
\begin{aligned}
& \operatorname{TDR}(l)=\sum_{i=1}^{M} R_{i, l}, l=\overline{1, M} \\
& T D R^{*}(l)=1-\frac{T D R(l)}{\max (\operatorname{TDR}(l))}
\end{aligned}
$$

The characterization of the transient signals was achieved through phase diagram representation in polar coordinates of the trajectory. It goes that the fundamental frequency of the transient signal is:

$$
T_{0}=\frac{2 n_{0}}{f_{s}}
$$

where $n_{0}$ is the number of points from the phase diagram between the angles $-3 \pi / 4$ and $\pi / 4$ and $f_{S}$ is the sampling frequency.

The characterization of the water hammer effect was realized using an ultrasonic sensing system placed on a pipe line. The acoustic effect induced to the sensors is detected using the recurrence plot analysis concept [4] (equation (1)) and, then, the time of flight of the signals arrived at both sensors is computed (figure 4). Hereby, the pressure variation performed by the water hammer phenomenon into the pipe line is determined and compared with the variation measured by the pressure sensor placed in the pipe. An error under $2 \%$ was obtained, the recurrence plot analysis method proving to be a much better approach in comparison to state of the art methods in hydraulics or signal processing.

Figure 4. Water hammer detection and localization using the TDR measure
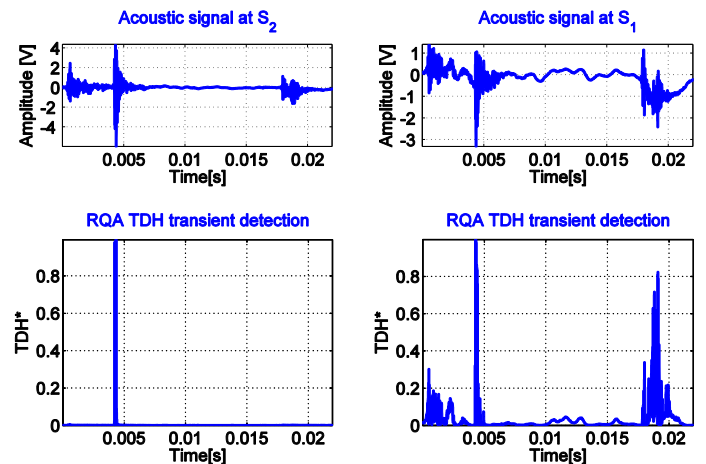

The detection and characterization of a rotational vortex rope using acoustic sensors in active configuration. The recorded signals are investigated through the quantification of the diagonal lines of the recurrence matrix (equation (3)) and the exploit of their frequency content. Results confirm the existence of the rotational vortex rope and highlight its evolution. The quantification is obtained using the measure:

$$
s d[n]=\frac{1}{M-n} \sum_{i=1}^{M-n} D\left(\overrightarrow{v_{i}}, \overrightarrow{v_{i+n}}\right), n=\overrightarrow{0, M-1}
$$

where $D\left(\overrightarrow{v_{i}}, \overrightarrow{v_{i+n}}\right)$ is the point $(i, j)$ corresponding to the distance matrix.

The applications concerning the electrical systems applications refer to the detection, localization and characterization of the electrical partial discharges measured in high voltage cables and in photovoltaic panels, respectively.

Figure 5. The area evolution of the phase space representations of the normalized multi-path acoustic signal and their corresponding phase diagrams
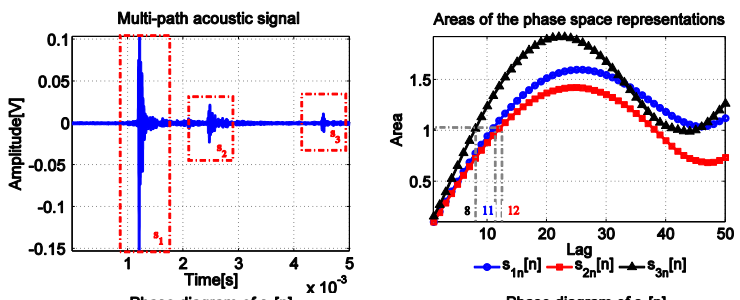

Phase diagram of $\mathrm{s}$,

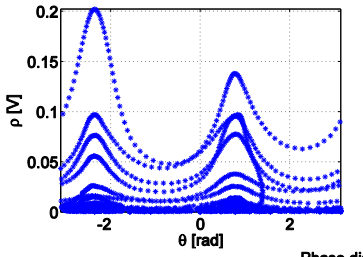

Phase dlagram of $s_{2}[n]$
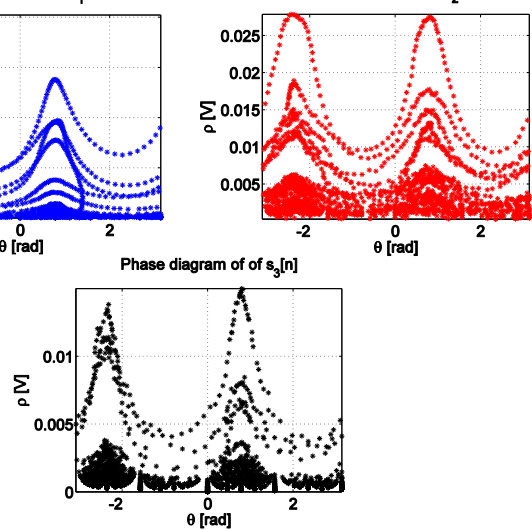

While the detection and the localization of the partial discharges are achieved through the exploration of the characteristics of the recurrence matrix building a well suited energy detector 
(equation (1)), their characterization is obtained using the multi-lag recurrence plot analysis, a new concept which uses the $2 \mathrm{D}$ phase space representation (figure $5)$.

The multi-lag recurrence plot analysis tools investigate the evolution of the signals in the phase space using the elliptic modeling and the polar coordinates representation (equation (3)) and highlights slight changes between the partial discharge and its reflexions.

\section{CONCLUSIONS}

Are our scientific activities in consensus with actual transitions from "the age of information to the age of (big) systems in engineering? Difficult to answer!

But, from twenty years we promote the idea that engineering's systems are nonlinear ones and usually can have chaotically behaviors and their associated processes need special studies' methods. And these ideas are in consensus with the worldwide research trends in communications and information technology.

\section{ACKNOWLEDGMENT}

The paper has been financially supported within the project entitled "Horizon 2020 - Doctoral and
Postdoctoral Studies: Promoting the National Interest through Excellence, Competitiveness and Responsibility in the Field of Romanian Fundamental and Applied Scientific Research", contract number POSDRU/159/1.5/S/140106. This project is cofinanced by European Social Fund through Sectoral Operational Programme for Human Resources Development 2007- 2013. Investing in people!

\section{REFERENCES}

[1] A.-V. Diaconu, and K. Loukhaoukha, "An improved secure image encryption algorithm based on Rubik's cube and digital chaotic cipher," Mathematical Problems in Engineering, vol. 2013, Article ID: 848392, 10 pages, 2013. doi: $10.1155 / 2013 / 848392$.

[2] A.-V. Diaconu, "An image encryption algorithm with a chaotic dynamical system based Sudoku Grid," in Proc. of the IEEE $10^{\text {th }}$ Int. Conf. on Communications, pp. 1-4, Bucharest, Romania, 29-31 May, 2014.

[3] A.-V. Diaconu, A. Costea, M. A. Costea, "Color image scrambling technique based on transposition of pixels between RGB channels using Knight's moving rules and digital chaotic map," Mathematical Problems in Engineering, vol. 2014, Article ID: 932875, 15 pages, 2014, doi: $10.1155 / 2014 / 932875$

[4] C. Ioana, A. Digulescu, A. Serbanescu, I. Candel, F. M. Birleanu, "Recent Advances in Non-stationary Signal Processing Based on the Concept of Recurrence Plot Analysis", Springer Proc. in Mathematics \& Statistics, M. Marwan et al. (Eds.): "Translational Recurrences", pp. 75-93, Cham, Switzerland, 2014. 